\title{
Math Contests: Solutions without Solving
}

\author{
Mārtiņš OPMANIS \\ Institute of Mathematics and Computer Science, University of Latvia \\ 29 Raina Boulevard, Riga, LV-1459, Latvia \\ e-mail:martins.opmanis@lumii.lv
}

\begin{abstract}
The paper gives an insight in the possibility to use tools and methods usually not allowed at mathematical olympiads and contests for finding correct answers for original problems. Lot of problem examples from the various math contests are given. Possible effects and risks of competition format change are discussed, caused by usage of additional tools and Internet resources.
\end{abstract}

Keywords. Olympiads in Informatics, Math contests, competition tasks, grading, online tools.

\section{Introduction}

There are lot of popular math contests or their rounds where just a short answer like some number (listed together with few other possible options or without them) must be provided instead of a full "classic" solution with an adequate level of reasoning. Among such contests are, for example, Kangaroo [Kangaroo], MAA American Mathematics Competitions - American Junior High School Mathematics Examination, AMC 8 [AJHSME], AMC 10, AMC 12 and AIME [AMC], Sri Lankan Mathematics Competition [SLMC], Schools Maths Olympics held by University of Melbourne [MUMS SMO] and others. The famous resource "Project Euler" which "is a series of challenging mathematical/computer programming problems that will require ... programming skills ... to solve most problems" [Project Euler] also requires single numbers as answers to problems.

In this sense "finding a correct answer" usually is used as a synonym to "properly solve the task in the usual way as consecutive steps of correct reasoning and conclusions". And most probably, the intention of problem setters is that there is practically impossible to guess the correct answer or find it in any other way, and the solving process must be completed anyway. And the only difference is that it is not required to describe this process in a "polished" form, but only the final result must be provided. Such "lightweight" competition format is appealing due to the fast and simple answer grading 
process. If additional tools are not used, then this assumption is almost correct, and the only way is to complete whole way from the given in a task statement till the correct answer, usually described in written form and named as "solution" and corresponding process as "solving". So we get "solution with solving".

Nowadays at the competitions in mathematics from the very basic level till the top - International Mathematics Olympiad (IMO) [IMO] only a limited number of tools are allowed in the process of problem solving. These tools are discussed in Section 2.

However, outside of competitions there are plenty of tools available which can help to find problem solutions. Some of them are described in Section 3.

With additional tools it is possible to obtain correct answers without the usual reasoning process sometimes essentially faster than in the "classical" way. Therefore "solution phase" (in its old meaning) may be omitted or, more correctly, a completely different way how to obtain the correct answer may be found. Further in this paper we will draw clear distinction between "solving" in its classical meaning and "finding correct answer" by using tools, methods, information sources, completely different from the solving process. In general, by "solution without solving" we denote the process of finding a correct answer by using an approach essentially different from the intended one. More precise description of this "alternative process" is given in Section 4.

By rise of computers programming in general became a powerful tool for rapid calculations and even for proving of propositions. As Petar S.Kenderov pointed out: "The nature of the mathematical research has changed significantly since considerable computing power came to the desk of almost every researcher and student. Mathematicians today can conduct complicated numerical experiments, use software for complex algebraic and analytic transformations, find patterns in huge data sets." [Kenderov]

By wide dissemination of computers and by the appearance of appropriate software (like as spreadsheets - MS Excel, Google Spreadsheets), appearing of Internet with enormously powerful information search capabilities (Google) and web-based tools (like as WolframAlpha [WA]), the possibility to find answers to particular mathematical problems increased dramatically. The main threat here is the possibility to obtain a correct answer having no clue about the solving process or even without understanding of the task statement.

Retaining of the old fashioned style of mathematical competitions makes bigger and bigger the breach between everyday life and math contests. Similarly, we could ask to perform communication tasks without usage of mobile phones and social networks, or to perform routine calculations without using electronic devices. At some point, such limitations becomes too orthodox and this may decrease the interest to participate in such competitions. This represents a serious problem also for problem setters - should avoid offering problems for which one can find straightforward solution in the "world of computer aided tools". For example, routine tasks like as multiplication of two long integers is not interesting as a contest problem.

At the same time Math olympiads are not alone in testing traditional mental skills. For example, at the Latvian national and Baltic regional level quite popular is a version of the Estonian mental calculation competition Miksike MentalMath[Miksike] where students are asked to perform fast calculations without additional tools. 
Further in Section 5, problems from various mathematics competitions will be analyzed. Problems described in the present paper may be considered as outcomes of the experiment "What could happen if at real math contests additional tools would be allowed?". Although most of the examples below come from various math competitions, similar problems are observed also in informatics(programming) competitions [IOI, $\mathrm{BOI}$ ] where solutions are mainly programs written in a programming language. The socalled "open input" tasks where problem solving strategy is not clearly defined are formally allowed but are rarely used. For solving problems in "Project Euler", it is intended that some programming must be involved. If correct answer will be found without programming, this also can serve as illustration of "solution without solving".

\section{Tools Allowed at Mathematical Contests}

Till now, in the classical mathematics competitions up to IMO only Euclidean tools straightedge and compass [MathWorld] allowed. These tools allow creating of the basic geometric constructions known since Euclid's "Elements". Without irony it can be noted that the same tools are used in math competitions for centuries (even if we start counting from the first typeset of these books in 1482). These two simple tools can illustrate the difference between tools used in classrooms and mathematics competition.

In the classroom, rulers (with centimeter or inches marks) of finite length are used - in opposite to math contests where straightedge has no marks and is assumed to possess infinite length. The story with compass is even more interesting. According to Euclid's third postulate "Given any straight line segment, a circle can be drawn having the segment as radius and one endpoint as center", compass still is quite a limited tool. More precisely - this tool is "collapsing compass" or "compass without memory" - $\mathrm{i}$. e. after drawing a circle, it loses any reference to the provided circle and the user must find a new segment and choose its endpoint to draw new circle. Quite a natural construction - drawing of two circles with the same radius with the centers at two given different points may not be performed by simple movement to another destination without changing the compass aperture. Luckily, Euclid himself (second proposition in Book I of "Elements") showed that also such compass can be used for transfer of distance to a given point and therefore its capacity is the same as the one of a "real" compass, and people must not worry about such limitations. Strictly speaking, when using a "real" compass, its equivalence to the abstract one must always be mentioned. Moreover, this is not case with straightedge and ruler (it is impossible to model ruler marks by simple straightedge).

\section{Tools Available}

One obvious tool which could be included in math contests is calculator, which became widely accessible for the general public since 1970-s. By the end of this decade 
calculators became common in schools. However, till now in a lot of competitions the usage of calculators is prohibited without excuses - IMO, AMC, FERMAT and Math Bowl [UT contests], Kettering Mathematics Olympiad [KMO], Schools Maths Olympics [MUMS SMO].

At the same time other competitions are not so strict - a limited use of calculators is allowed in contests organized by University of Waterloo: "Calculators are allowed, with the following restriction: you may not use a device that has internet access, that can communicate with other devices, or that contains previously stored information. For example, you may not use a smartphone or a tablet." and (for Contests with Full Solution Questions): „While calculators may be used for numerical calculations, other mathematical steps must be shown and justified in your written solutions and specific marks may be allocated for these steps. For example, while your calculator might be able to find the $x$-intercepts of the graph of an equation like $y=x^{3}-x$, you should show the algebraic steps that you used to find these numbers, rather than simply writing these numbers down." [Waterloo]

In Australian Mathematical Competition, calculators are banned starting from the year 7 and are allowed for younger participants as one of "aids normally available to them in the classroom" [Australian MC]. In the Math competition for students who are deaf or hard of hearing organized by Rochester Institute of Technology, only calculators with four basic arithmetic operations and square, square root and percent calculations are allowed. Scientific and graphing calculators are not permitted [RIT competition].

However, there are competition focused on usage of calculators - Calculator Applications Contest [CAC] or particular rounds of MATHCOUNTS Competition [MATHCOUNTS] and PA Math League and Atlantic Pacific Math League Contests [PAML].

Completely different "tool" which could be mentioned are sheets of limited number of formulas distributed for secondary school students at final exams in mathematics in Latvia. Similar supporting material are books together with printed materials of limited volume which teams are allowed to prepare in advance in secondary school students team competition in programming VKOSHP[VKOSHP].

Today, Internet together with personal computers, tablets, smartphones and other electronic devices may be qualified as a "tool available for secondary school students".

There are several competitions designed especially by assuming the free usage of Internet resources and making sometimes impossible to solve tasks without additional resources. Characteristic representatives of such competitions are The Internet Problem Solving Contest[IPSC] and MIT Mystery Hunt[MIT], there are a lot of competitions aimed at finding facts in the Internet.

There are only a few contests and competitions where the usage of tools and Internet resources is allowed but not always required for finding correct answer. Project Euler [Euler] and Latvian team competition in Mathematics and Informatics "Ugāle" [Opmanis] are among the few which can be named.

An interesting topic would be the classification of problem statements, solving strategies, etc. at the various existing competitions, but this is out of the scope of the present paper. 


\section{Description of "Finding Correct Answer"}

It is very easy to argue against usage of Internet or sophisticated electronic devices: "You always may ask for help from aside by using Skype or e-mail and nobody can be sure that these answers are obtained you at all."

If we look from the perspective of a problem setter, several simple "fair play" rules must be stated:

1. No help from outside is allowed - no other people may be involved in the process of finding the answer directly or indirectly (like direct conversation or asking for advice in a forum or a newsgroup).

2. Only free, accessible for all, resources and tools may be used.

3. The goal is to speed up process of finding correct answer - i. e. the total time of finding answer must be less if compared with the classical approach where no additional tools are involved.

4. It shouldn't be possible to find a correct answer simply by using the task attribution. For example, by searching for an answer or solution of a particular competition in some published source because the title of competition or book is known. As an example here may serve IMO and its satellite resource - special section in website "Art of Problem Solving" [AoPS]. Solutions of "Project Euler" [Project Euler] tasks are also widely discussed on the Internet, so knowing that some particular task comes from the project, you will find a lot of hints, program examples or even correct answer.

5. Tasks must be non-trivial and non-routine, different from the well known tasks like "find the least possible Pythagorean triangle" or "calculate the fifth Fibonacci number". There must be no specialized tools (websites) for solving of general tasks of this class. For example, for tasks where a particular member of series must be found, an excellent resource is The On-Line Encyclopedia of Integer Sequences ${ }^{\circledR}$ founded by N.J.A.Sloane [OEIS $\left.{ }^{\circledR}\right]$ - if few first members of sequence are provided, all the possible candidates together with vivid supporting information and references are reported back.

6. Clairvoyance like "I just saw the answer in my mind and can't explain why it is correct." or lucky guessing is not counted. Process must be repeatable and determined.

7. And the last but not least - there MUST exist verifiable correct answer of the task. For example, tasks like "What is the next integer in the sequence $2,0,2,1,2,2,2,2,2,2,3,2,4, \ldots$ ?" doesn't belong to this class, because without additional comments it is impossible to prove that the answer is correct (even if there exists a simple deterministic algorithm as in the above example).

Some remarks may be added:

As in the classical problem solving, impossibility to find an appropriate answer doesn't guarantee that answer cannot be found in a different way, by different tool or by different people. 
There must be gained some experience to get maximum profit from the available tools and to see that some resource may be helpful. Even when final answer can't be found directly, usage of tools may help in finding new ideas or ways how the problem may be solved.

English (in general - any "big" language) speakers have advantage due to a larger number of useful resources.

From the problem setter's perspective, if almost unlimited usage of tools is allowed as described above, the invention of new tasks becomes a real challenge. Even if the task inventor is confident that the task is created by himself, "stress test" must be provided to be sure that there are no obvious workarounds or alternative approaches that could making the finding of the correct answer too easy. From the other side - if usage of some tools is assumed, the task may become too hard for solvers not familiar with particular tool and therefore the task becomes too narrow-focused.

Alternative way how to use old tasks is to "obfuscate" the original formulation so that keywords cannot be used in search engines to get on right track. Or "wrap" simple equations in the form of text problems and force contestants to correctly "unwrap" the task before solving.

\section{Finding Answers for Tasks from the Previous Math Contests}

To justify the approach with almost unrestricted usage of additional tools allowed, a lot of problems (tasks) from the previous math contests of various levels were investigated. This class of tasks is chosen because: 1) they are short in form, 2) they are clear and selfcontained (no additional knowledge like foreign language is necessary to comprehend the task statement), 3) there is a unique answer. Of course, these tasks are not designed as tool usage exercises and some of them were created even before computers became widely used. These examples represent just a brief sketch of what are the options today if we want to obtain correct answers without solving. Original task formulations are given below together with competition title and problem number, brief description of tool(s) used, and how the usage of these tools helped in finding correct answer. Examples are denoted by "E" followed by the consecutive example number.

E1. USA AMC 8 1999, Problem 24

When $1999^{2000}$ is divided by 5, the remainder is
(A) 4
(B) 3
(C) 2
(D) 1
(E) 0

Tool: WA. Input expression " $1999 \wedge 2000 \bmod 5$ " and obtain the answer 1 immediately. Therefore the correct answer is answer D. Only basic knowledge about expression syntax in WA is necessary. Almost the same approach works for USA AJHSME 1996, task 15 .

E2. USA AMC 8 2000, Problem 14

What is the unit digit of $19^{19}+99^{99}$ ?
(A) 0
(B) 1
(C) 2
(D) 8
(E) 9 
Tool: WA. Input expression " $19^{\wedge} 19+99^{\wedge} 99$ " and obtain a 198 digit integer with the last digit 8 . Therefore the correct answer is answer D. Only basic knowledge about expression syntax in WA is necessary.

E3. USA AMC 8 2010, Problem 24
What is the correct ordering of the three numbers, $10^{8}$, $5^{12}$, and $2^{24}$ ?
(A) $2^{24}<10^{8}<5^{12}$
(E) $10^{8}<2^{24}<5^{12}$
(B) $2^{24}<5^{12}<10^{8}$
(C) $5^{12}<2^{24}<10^{8}$
(D) $10^{8}<5^{12}<2^{24}$

Tool: WA. Step 1: Input expression " $10^{\wedge} 8-5 \wedge 12$ " and obtain the answer -144140625 . Therefore $10^{8}<5^{12}$ and possible valid candidates are just (A), (D) and (E).

Step 2: Input expression " $10^{\wedge} 8-2^{\wedge} 24$ " and obtain the answer 83222784 . Therefore $10^{8}>2^{24}$ and the correct answer is answer (A). It was just coincidence that the correct answer was obtained by two queries. However, even if more queries would be used, it is still possible to obtain the correct answer without any clue about comparison of powers in general. Only basic knowledge about expression syntax in WA and simple logical reasoning is necessary.

E4. USA AMC 8 2011, Problem 24

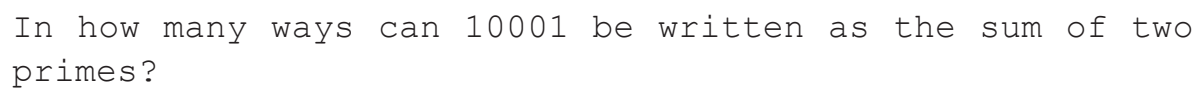

Tool: WA. Input query EXACTLY as it is formulated "In how many ways can 10001 be written as the sum of two primes?". Despite the fact that WA responds "WA doesn't understand your query", it gives valuable information about the number 10001 itself and theoretically from "10001 is an odd number." you can derive a correct answer. If this doesn't help, the initial textual query may be modified: "10001 as the sum of two primes". Now, WA is processing this query and gives the result "no such prime numbers exists". Therefore, the answer (A) is the correct one. It must be emphasized that the only skill necessary was a correct rephrasing of query. Even the knowledge of what does "prime number" means was not necessary at all.

E5. USA AJHSME 1995, Problem 3
Which of the following operations has the same effect on a number as multiplying by $3 / 4$ and then dividing by 3/5? (A) dividing by $4 / 3$ (B) dividing by $9 / 20$ (C) multiplying by $9 / 20$ (D) dividing by $5 / 4$ (E) multiplying by $5 / 4$

Tool: WA. Input the expression " $a *(3 / 4) /(3 / 5)$ " and obtain the result " $5 a / 4$ ", from which you can decide that $(\mathrm{E})$ is the correct answer. In this query a general variable "a" is used. However, even without it you can obtain the answer $5 / 4$ and by using some basic knowledge can transform it to a correct test answer. 
E6. USA AJHSME 1995, Problem 15

What is the $100^{\text {th }}$ digit to the right of the decimal point in the decimal form of 4/37?
(A) 0
(B) 1
(C) 2
(D) 7
(E) 8

Tool: WA. Input the expression “ (floor $(4 / 37) \star 10 \wedge 100)) \bmod 10^{\prime}$ " and obtain the result 1 , from which you can decide that (B) is the correct answer. Skills necessary to formulate a correct query are above the basic level and you must know how to use arithmetical functions. However, if you know that, you need not to know anything about the character of infinite decimal fractions like 4/37 - just translate the query and obtain the answer.

E7. International Mathematical Talent Search [IMTS] - Round 9 - Problem 4
A triangle is called Heronian if its sides and area are integers. Determine all five Heronian triangles whose perimeter is numerically the same as its area.

Tool: Google search for "Heronian triangle". One of the first resources is Wikipedia topic [http://en.wikipedia.org/wiki/Heronian_triangle] having special paragraph „Equable triangles”, where it is said: „A shape is called equable if its area equals its perimeter. There are exactly five equable Heronian triangles: the ones with side lengths $(5,12,13),(6,8,10),(6,25,29),(7,15,20)$, and $(9,10,17) . "$ So you can obtain the correct answer without any calculations, reasoning, etc. Key to the success was a mathematically correct naming of terms in the problem statement and careful reading of source found. If „Heronian” would be replaced by other word, the described approach would not work. Of course, from the description of „Equable triangles" you must be wise enough to understand that these are the triangles you are searching for.

E8. Project Euler - Problem 21 „Amicable numbers”

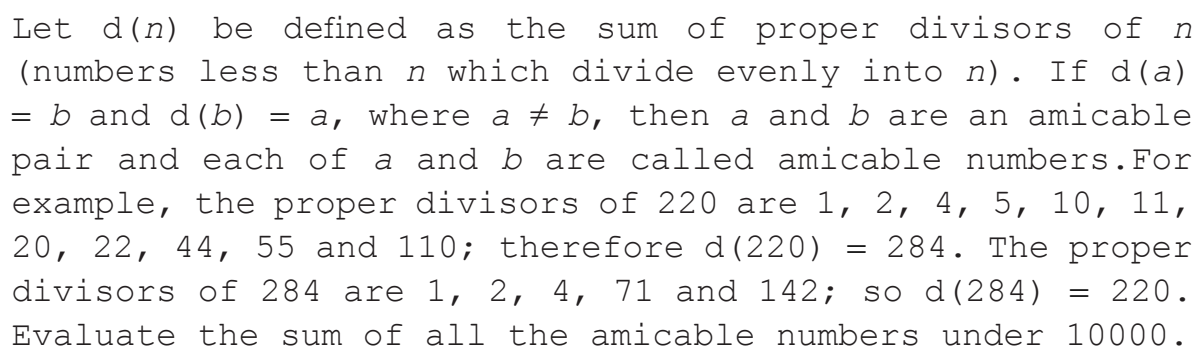

Tool: Google search for "Amicable numbers". One of the top resources is a reference to sequence A063990 [OEIS]. In this resource, the first members of the sequence of amicable numbers are listed: 220 , 284, 1184, 1210, 2620, 2924, 5020, 5564, $6232,6368,10744,10856, \ldots$ 
You can observe that only the first ten of them are under 10000 and you can simply sum them up obtain the correct answer. Essential again was a consequent usage of the term ,amicable numbers”. Otherwise, it would be impossible to obtain the answer so quickly, or (in case if this term would be used for denoting different concept) the answer would be incorrect.

E9. Project Euler - Problem 19 „Counting Sundays”

You are given the following information, but you may prefer to do some research for yourself.

- 1 Jan 1900 was a Monday.

- Thirty days has September, April, June and November.

All the rest have thirty-one, Saving February alone, Which has twenty-eight, rain or shine. And on leap years, twenty-nine.

- A leap year occurs on any year evenly divisible by 4, but not on a century unless it is divisible by 400 .

How many Sundays fell on the first of the month during the twentieth century (1 Jan 1901 to 31 Dec 2000)?

Tool: An old notebook was received as a gift during the $9^{\text {th }}$ Baltic Olympiad in Informatics 2003 in Estonia (in Estonian!) containing the so-called calendar of the century (fragment is shown in the Fig. 1).

Even without any knowledge of Estonian language it is possible to guess that 0 in the table denotes that the first day in the chosen month of a particular year is Sunday. For example, 1st of October of 1961 was Sunday. To get correct answer it is enough to process necessary rows and calculate total number of zeroes.

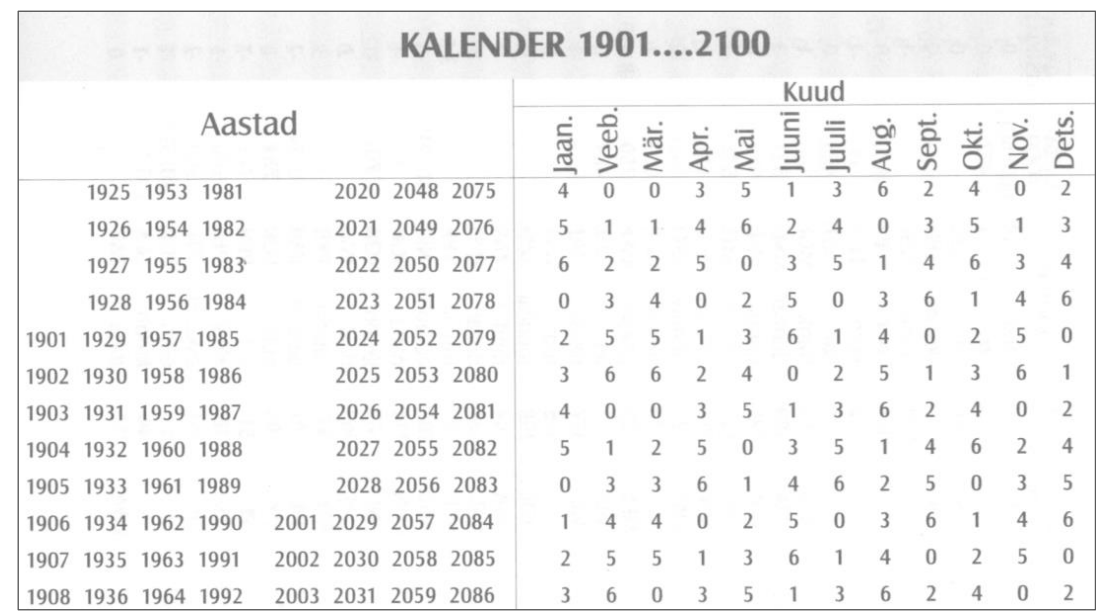

Fig. 1. Calendar of Centuries - Estonian version. 


\section{E10.}

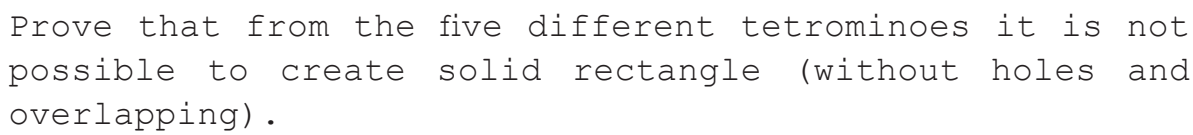

Prove that from the five different tetrominoes it is not possible to create solid rectangle (without holes and overlapping).

Tool. This may be called ,proof found by accident”, because when searching for „Tetromino", as one of top results you obtain Wikipedia page with a brief proof of the stated problem (second picture at the right hand side).

E11. British Mathematical Olympiad 2007 - Problem 2.

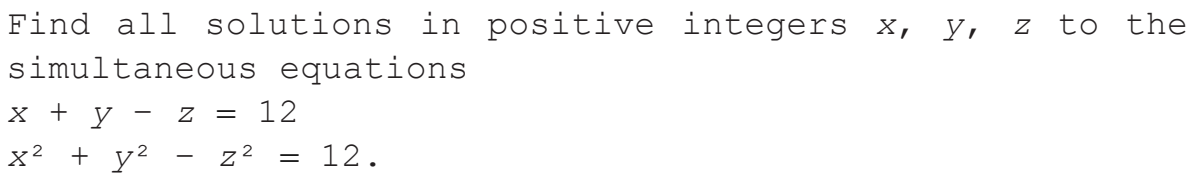

Tool. WA. Input “ $x+y-z=12, \quad x^{\wedge} 2+y^{\wedge} 2-z^{\wedge} 2=12, \quad x>0, \quad y>0, \quad z>0$ ” and obtain eight possible answers $(\mathrm{x}, \mathrm{y}, \mathrm{z}):(13,78,79),(14,45,47),(15,34,37)$, $(18,23,29),(23,18,29),(34,15,37),(45,14,47),(78,13,79)$. The only necessary skill was correct reformulation of initial problem in WA syntax. On this particular example, two side effects could be observed: a) one can easy write incorrect equations just by omitting comma separating both equations. This leads to misinterpretation of equations and wrong answers, b) WA is evolving and the interpretation of an incorrect set of equation in different tool versions may change over the time.

For this particular problem, the input " $x+y-z=12 x^{2}+y^{2}-z^{2}=12$ " in different WA versions was interpreted as " $x+y-z=12,12 x^{2}+y^{2}-z^{2}=12$ ” (lot of ,valid" integer answers having nothing in common with the solutions of the original equations) or , $x+$ $y-z=12 x^{2}+y^{2}-z^{2}$ " (no solutions in integers).

E12. International Mathematical Talent Search Round 10 - Problem 1.

Find $x^{2}+y^{2}+z^{2}$ if $x, y$ and $z$ are positive integers such

that $7 x^{2}-3 y^{2}+4 z^{2}=8$ and $16 x^{2}-7 y^{2}+9 z^{2}=-3$.

Tool. WA. Input “ $\mathrm{p}=\mathrm{x}^{\wedge} 2+\mathrm{y}^{\wedge} 2+\mathrm{z}^{\wedge} 2, \mathrm{x}>0, \mathrm{y}>0, \mathrm{z}>0,7 \mathrm{x}^{\wedge} 2-3 \mathrm{y}^{\wedge} 2+4 \mathrm{z}^{\wedge} 2=8$, $16 x^{\wedge} 2-7 y^{\wedge} 2+9 z^{\wedge} 2=-3$ ” and obtain the answer $p=165, x=4, y=10, z=7$ immediately. It must be pointed out that in the input set of directives integers are not required.

E13. British Mathematical Olympiad 2007 - Problem 1

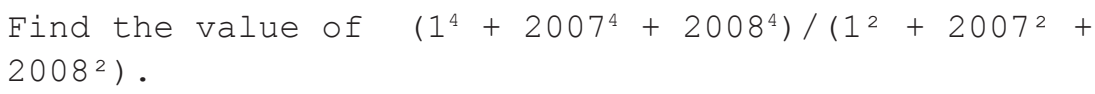

Tool. WA. Expression in almost original form may be given as input: ,$\left(1^{\wedge} 4+2007^{\wedge} 4+2008^{\wedge} 4\right) /\left(1^{\wedge} 2+2007^{\wedge} 2+2008^{\wedge} 2\right) "$ and answer 4030057 is obtained straightforwardly 
E14. Pan African Mathematical Competition 2004 - Problem 2

Is 4 sqrt $(4-2$ sqrt(3)) + $\operatorname{sqrt}(97-56$ sqrt(3)) an integer?

Tool. WA. Simple "Copy-Paste" from the original problem formulation into WA and as a result the value 3 is obtained. So, answer to the original question is "yes".

E15. British Mathematical Olympiad 2001 - Problem 1

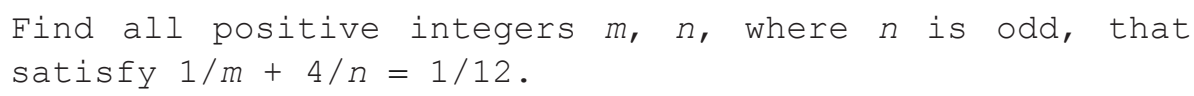

Tool. WA. Query must be slightly modified to fulfill the " $n$ is odd" requirement: "solve $\{1 / \mathrm{m}+4 / \mathrm{n}=1 / 12, \mathrm{n}=2 \mathrm{k}+1, \mathrm{~m}>0, \mathrm{n}>0\}$ over the integers". Execution gives three pairs of answers $(\mathrm{n}, \mathrm{m}):(49,588),(51,204)$ and $(57,76)$.

E16. 1960 IMO - Problem 1

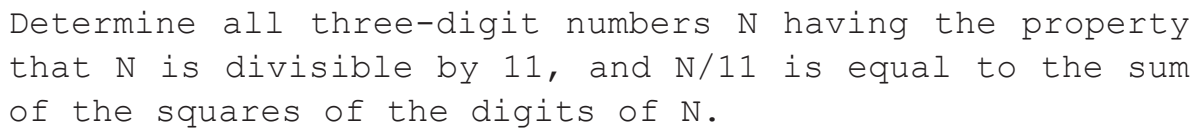

Tool. WA. Nontrivial translation of the original statement in terms of WA is necessary. The corresponding query is "solve $\left(100 a+10 b+c-11\left(a^{\wedge} 2+b^{\wedge} 2+c^{\wedge} 2\right)=0\right.$; $\mathrm{a}>0, \mathrm{~b}>=0, \mathrm{c}>=0)$ over the integers". Two answers $(\mathrm{a}, \mathrm{b}, \mathrm{c}):(5,5,0)$ and $(8,0,3)$, which must be interpreted as three-digit numbers 550 and 803 . You may consult the resource [AoPS] to see that these answers are the same as in the official solution.

E17. 1962 IMO - Problem 4

Solve the equation $\cos ^{2} x+\cos ^{2} 2 x+\cos ^{2} 3 x=1$

Tool. WA. Input query "solve $\left(\cos (x)^{\wedge} 2+\cos (2 x)^{\wedge} 2+\cos (3 x)^{\wedge} 2=1\right)$ " and get answer: $x=(8 \pi n+\pi) / 4, x=\pi(8 n-1) / 4, x=\pi(8 n-3) / 4, x=\pi(8 n+3) / 4, x=$ $\pi(4 \mathrm{n}-1) / 2$, where $\mathrm{n} \in \mathrm{Z}$.

E18. 1962 IMO - Problem 2

Determine all real numbers $x$ which satisfy the inequality: $\sqrt{ }(\sqrt{ }(3-x)-\sqrt{ }(x+1))>1 / 2$

Tool. WA. The query "solve ( sqrt ( $\operatorname{sqrt}(3-x)-\operatorname{sqrt}(x+1))>1 / 2)$ " gives straight answer $-1 \leq x<(32-\sqrt{ } 127) / 32$.

E19. 1965 IMO - Problem 4

Find all sets of four real numbers $x_{1}, x_{2}, x_{3}, x_{4}$ such that the sum of any one and the product of the other three is equal to 2 . 


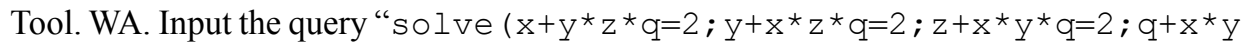
${ }^{\star} \mathrm{z}=2$ ) over the real numbers" and obtain the answers shown in Fig. 2.

Surprisingly, a simplified query "solve $\left(x+y * z * q=2 ; y+x * z * q=2 ; z+x{ }^{\star} y^{\star} q=\right.$ $\left.2 ; q+x^{\star} y^{\star} z=2\right)$ " gives more appealing values of $(x, y, z, q)$ (however, they are tailed with complex values and you must be able to distinguish them): $(-1,-1,3,-1)$, $(-1,3,-1,-1),(3,-1,-1,-1),(1,1,1,1)$ and $(-1,-1,-1,3)$.

The same answers could be obtained by substitution in the expressions for $\mathrm{z}$ in Fig.2.

E20. International Mathematical Talent Search [IMTS]- Round 44- Problem 2

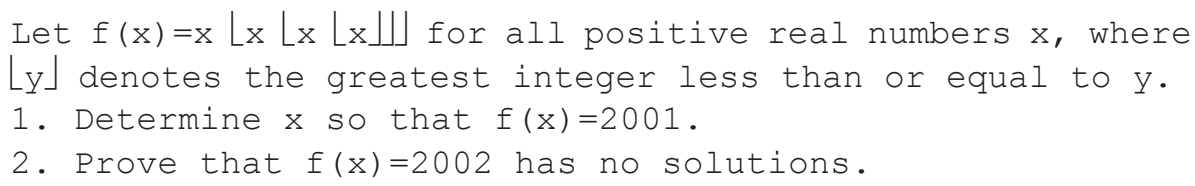

Let's split this problem into two sub-problems - (1) and (2) and as the first step let's try to find an answer for the sub-problem 1.

Tool: WA. Execution of the query " $x{ }^{\star}$ floor $\left(x^{\star}\right.$ floor $\left(x^{\star}\right.$ floor $\left.\left.(x)\right)\right)=2001$ " gives the answer $x=2001 / 286$.

To solve E20 spreadsheets may be used as well. Let's denote $\mathrm{p}=$ floor( $\mathrm{x}$ floor( $\mathrm{x}$ floor $(\mathrm{x}))$ ). Then the solution of equation $\mathrm{x}=2001 / \mathrm{p}$ must be found. We can use the exhaustive search by filling six columns of spreadsheet with values of $\mathrm{p}$ (by definition, $\mathrm{p}$ is integer), $\mathrm{x}$, floor(x), floor(x floor(x)), floor(x floor(x floor(x)) ) and floor(x floor(x floor(x)))-p. It is easy to go through all the values from 1 to 2001 looking for a value of $\mathrm{p}$ leading to 0 in the sixth column. Excerpt of the spreadsheet is given in the Fig. 3.

Let's consider solving of the second sub-problem of E20 by using the same approach as in the first sub-problem.

Tool: WA. Execution of the query " $x *$ floor $(x *$ floor $(x *$ floor $(x)))=2002$ " gives the answer $\mathrm{x}=7$ (with the remark that standard computation time exceeded). This answer is incorrect, because $7^{4}=2401 \neq 2002$.

Spreadsheet approach is better. Excerpt is given in the Fig. 4.

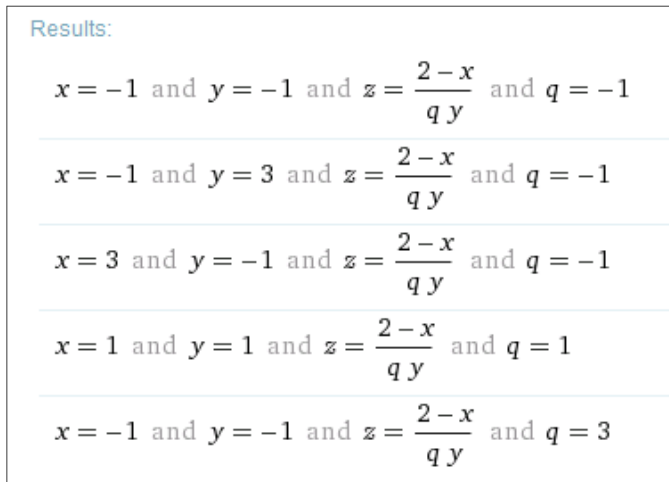

Fig. 2. Results of query for E19. 


\begin{tabular}{|c|c|c|c|c|c|}
\hline$p$ & $x=2001 / p$ & floor $(x)$ & floor $(x \cdot$ floor $(x))$ & $\begin{array}{l}\text { floor }( \\
x \cdot \text { floor }(x \cdot \text { floor }(x))\end{array}$ & $\begin{array}{l}\text { floor }( \\
x \cdot \text { floor }(x \cdot \text { floor }(x)) \\
\qquad)-p\end{array}$ \\
\hline 283 & 7,070671378 & 7 & 49 & 346 & 63 \\
\hline 284 & 7,045774648 & 7 & 49 & 345 & 61 \\
\hline 285 & 7,021052632 & 7 & 49 & 344 & 59 \\
\hline 286 & 6,996503497 & 6 & 41 & 286 & 0 \\
\hline 287 & 6,972125436 & 6 & 41 & 285 & -2 \\
\hline 288 & 6,947916667 & 6 & 41 & 284 & -4 \\
\hline 289 & 6,923875433 & 6 & 41 & 283 & -6 \\
\hline
\end{tabular}

Fig. 3 Excerpt from the Google spreadsheet for finding answer of the first sub-problem of E20 (formulas are shown as column headers).

\begin{tabular}{|c|c|c|c|c|c|}
\hline$p$ & $x=2002 / p$ & floor $(x)$ & floor $(x \cdot f$ loor $(x))$ & $\begin{array}{l}\text { floor }( \\
x \cdot \text { floor }(x \cdot f \operatorname{loor}(x)) \\
,\end{array}$ & $\begin{array}{l}\text { floor( } \\
x \cdot \text { floor }(x \cdot f \operatorname{floor}(x)) \\
\quad,-p\end{array}$ \\
\hline 285 & 7,024561404 & 7 & 49 & 344 & 59 \\
\hline 286 & 7 & 7 & 49 & 343 & 57 \\
\hline 287 & 6,975609756 & 6 & 41 & 286 & -1 \\
\hline 288 & 6,951388889 & 6 & 41 & 285 & -3 \\
\hline
\end{tabular}

Fig. 4 Excerpt from the Google spreadsheet for finding answer of the second sub-problem of E20 (formulas are shown as column headers).

From the spreadsheet you can conclude that there is no solution - a value of $\mathrm{p}$ for which difference in the sixth column is 0 .

Are the above examples "good examples" (in almost all cases we succeed in finding a correct answer) or "bad examples" (in some cases it was too easy to obtain the right answer without proper evidence of adequate thinking process)? It depends whether you are a problem setter and are worried about the possibility of cheating by using modern gadgets with Internet access, or just want to check your solution of the task, or represent different group of people.

Will the future be so simple? Just input your equation and obtain a correct answer without any clue and without the need to understand how it works? We already observed problems with WA when tried to find answer to the sub-problem 2 of E20. Is this just an accident? Unfortunately, NO.

\section{E21.}

Find all solutions in integers of equation $(x+y)^{2}=x^{3}-y^{5}$.

In the original problem statement it was necessary to find all solutions in prime numbers. Obviously this would be a subset of the problem stated there.

Tool: WA. Input the equation “ $(x+y)^{\wedge} 2=x^{\wedge} 3-y^{\wedge} 5$ ” and you will obtain two solutions in integers $(x, y):(0,-1)$ and $(0,0)$. However, there exists at least one more obvious solution: $(1,0)$ not reported there. Even worse, there is a nontrivial answer $(7,3)$ not 
given without any sign that the answers found are not all possible or warning that it was not possible to calculate all solutions due to lack of resources or any other reason.

The general observation is as follows: "Tools may be not perfect and their produced answers may be incomplete or wrong even if you use them properly". For complicated tools like WA it is hardly possible to justify that tool is working properly for ALL correct inputs. If tools are used, you always must to justify the answers found. However, this justification task may as complicated as solving of the initial problem.

\section{Conclusions}

Computer and Internet based tools are very powerful and may be successfully used for finding answers for different math problems. However, their unlimited usage may be dangerous in a sense that young people may use them without necessary criticism like today calculators are used for routine calculations ignoring the possibility of computational errors. Tasks from the previous contests can't be given in such contests without careful examination as well. In the case when tools and resources are allowed a shift in trained skills is necessary - more attention must be paid to proper selection of the most appropriate tool, its usage and validation of the received answers. If we observe traditional topics in math contests - Algebra, Number theory, Combinatory and Geometry, it seems that problems from algebra and number theory are more suitable for tool usage in comparison with problems from geometry.

More tools, their usage for different problem classes and competitions, possible side effects and drawbacks must be further investigated.

\section{Acknowledgements}

I would like to thank Prof. Kārlis Podnieks for valuable comments.

\section{References}

AMC. Mathematical Association of America (MAA) American Mathematics Competitions.

http: //www.maa.org/math-competitions/amc-contests

AJHSME. American Junior High School Mathematics Examination.

http://artofproblemsolving.com/wiki/index.php/AMC_8_Problems_and_Solutions

AoPS. Art of Problem Solving. Community-Contest collections-IMO.

http: //artofproblemsolving.com/community/c3222_imo

Australian MC. Australiam Mathematics Competition. http://www. āmt. edu. au/amcfact.html

CAC. Calculator Applications Contest.

http://www.uiltexas.org/academics/calculator-applications

IMO. International Mathematics Olympiad. http: / / www . imo-official .org/

IMTS. International Mathematical Talent Search.

https://cms.math.ca/Competitions/IMTS, https://cms.math.ca/Concours/IMTS/

IPSC. The Internet Problem Solving Contest. http://ipsc.ksp.sk/ 
Kangaroo. International Mathematical Kangaroo, Kangourou sans frontières. http://en.wikipedia.org/wiki/Mathematical_Kangaroo

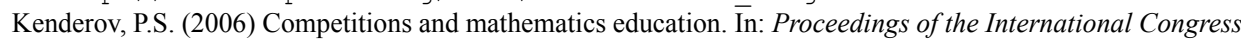
of Mathematicians, Madrid, Spain, 2006. 1583-1598.

KMO. Kettering Mathematics Olympiad. http://paws . kettering . edu// acheng/Olympiad/

MATHCOUNTS. Competition. http://www.artofproblemsolving.com/Wiki/index.php/MATHCOUNTS

MathWorld. http://mathworld.wolfram.com/EuclideanTools.html

Miksike. MentalMath. http://en.wikipedia.org/wiki/Miksike_MentalMath

MIT. Mystery Hunt. http: //www. mit.edu/ puzzle/

MUMS SMO. Schools Maths Olympics, Melbourne University Mathematics and Statistics Society. http://www.ms. unimelb. edu. au/ mums/olympics/smo.html

OEIS ${ }$. The On-Line Encyclopedia of Integer Sequences ${ }^{\circledR}$. https : / / oe is . org/

PAML. PA Math League and Atlantic Pacific Math League Contests. http: //www. npenn.org/page/1437

Project Euler. https: / / projecteuler. net/

SLMC. Sri Lankan Mathematics Competition. http: / / www.slmathsolympiad.org/slmcPast.jsp

Opmanis, M. (2009). Team competition in mathematics and informatics "Ugāle" - finding new task types. Olympiads in Informatics. 3, 80-100.

RIT competition. http://www.rit.edu/ntid/mathcompetition/what-kind-calculator-allowed

UT contests - FERMAT (Fundamental Exams of Remarkable Mathematical Ability and Talent) and Math Bowl. http: //www. math.utk. edu/MathContest/ContestInfo.html

VKOSHP. http://neerc.ifmo.ru/school/russia-team/rules.html

Waterloo. University of Waterloo, Mathematics and Computing Contests. http://www. cemc. uwaterloo.ca/contests/calculators.html

WA. WolframAlpha. http://www.wolframalpha.com/

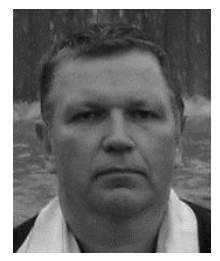

M. Opmanis is researcher at the Institute of Mathematics and Computer Science of University of Latvia. He is one of the main organizers of Latvian Olympiad in Informatics, was deputy or team leader of Latvian IOI and Baltic OI teams. M.Opmanis was head of jury of Baltic Olympiad in Informatics at BOI 1996, 1999, 2004 and 2012. Since 2012 he is member of IOI International Committee. 